\title{
B-04
}

\section{SEGUIMIENTO DE LA EVOLUCIÓN DE LOS NIVELES DE SALINIDAD Y NITRATOS EN EL ACUÍFERO SUPERFICIAL DE ZAFARRAYA Y EN FINCAS DE HORTICOLAS AL AIRE LIBRE}

\author{
Alonso López, F., Contreras París, J.I., Cánovas Fernández, G., González Vizcaino, A.
}

Instituto de Investigación y Formación Agraria y Pesquera de Andalucía (IFAPA), Centro La Mojonera, Camino San Nicolás, n¹, 04745 La Mojonera, Almería.

f.alonso@juntadeandalucia.es

\section{Resumen}

La horticultura al aire libre del Llano de Zafarraya es el motor económico de la comarca. El riego de este sector se sustenta en el sistema acuífero del Polje de Zafarraya y Sierra Gorda. En estos acuíferos, como consecuencia de la explotación agrícola de la zona, se viene produciendo desde hace décadas sobreexplotación del recurso, con la consiguiente salinización y nitrificación. Los objetivos de este trabajo han sido estudiar los niveles de salinidad y nitratos de las aguas del acuífero de Zafarraya y hacer un seguimiento de la solución del suelo en explotaciones hortícolas de la zona, para poder realizar recomendaciones que permitan mejorar la gestión de la fertilización y evitar el aumento de la contaminación por nitratos. Los niveles de salinidad obtenidos a lo largo del periodo de estudio han sido estables y no representan problemas para los principales cultivos hortícolas de la zona. En lo que respecta a la concentración de nitratos, los resultados muestran niveles excesivos por encima de $300 \mathrm{mg} \mathrm{L}^{-1}$. Estos resultados son consecuencia de la práctica habitual de aplicaciones excesivas de fertilizantes nitrogenados. La recomendación inicial sería mantener una concentración de nitratos estable a lo largo del ciclo de cultivo para evitar acumulaciones en el suelo sobre todo al final del mismo.

\section{Introducción y objetivos}

El Llano de Zafarraya, está situado al suroeste de la provincia de Granada en el límite con la de Málaga. Su especial microclima y la fertilidad de su suelo, la convierten en una zona de intensa actividad agrícola. La superficie de cultivo de unas 2000 hectáreas, está dedicada mayoritariamente al cultivo de hortícolas al aire libre (tomate, alcachofa, lechuga, coliflor, judía, etc.). El riego de este sector se sustenta en el acuífero detrítico del Polje y en el acuífero carbonatado subyacente de Sierra Gorda (Cherif et al. 1995).

Como consecuencia de la explotación agrícola de esta zona, se viene provocando una fuerte contaminación por nitratos del sistema de acuíferos. Ya en el año 1995, Morell y colaboradores cifraban niveles máximos de nitratos de $400 \mathrm{mg} \mathrm{L}^{-1}$ en los pozos del acuífero del Polje y, a día de hoy, en determinadas épocas son superiores a esta cantidad.

A pesar de la sobreexplotación del acuífero y el empeoramiento de la calidad del agua, la aplicación del riego y los fertilizantes realizados en la zona se basan fundamentalmente en la experiencia del agricultor. Esto supone, en muchos casos, la aplicación de dosis de agua y nutrientes excesivos.

La Unión Europea publicó la directiva 96/6767CEE relativa a la protección de las aguas contra la contaminación causada por nitratos procedentes de fuentes agrarias, con el 
fin de proteger la salud humana, los recursos vivos y los ecosistemas acuáticos. Para tal fin, se estableció un límite máximo de $50 \mathrm{mg}$ de nitratos por litro. Con arreglo a este valor en aguas superficiales o subterráneas, se han establecido 24 zonas vulnerables a la contaminación por nitratos en Andalucía (decreto 36/2008, de 5 de febrero, de la Junta de Andalucía y órdenes posteriores). Sierra Gorda-Zafarraya constituyen la zona vulnerable número 20. Para estas zonas se establece un código de buenas prácticas agrícolas y un programa de actuación de obligado cumplimiento (Orden 18 noviembre de 2008, Junta de Andalucía).

En este contexto, los objetivos de este trabajo han sido estudiar la evolución de los niveles de salinidad y nitratos de las aguas del acuífero superficial de Zafarraya y hacer un seguimiento de la solución del suelo en explotaciones hortícolas de la zona, con el fin de realizar recomendaciones que permitan mejorar la gestión de la fertilización y evitar el aumento de la contaminación por nitratos.

\section{Materiales y métodos}

El Polje de Zafarraya es una depresión interna endorréica que ocupa una extensión aproximada de $28 \mathrm{Km}^{2}$ y una altura media de $900 \mathrm{~m}$ sobre el nivel del mar. Presenta un clima mediterráneo semicontinental de media montaña (Gómez-Zotano et al. 2015) y la precipitación media anual es de 950 mm (ITGE-JUNTA, 1998). El acuífero detrítico del Polje, es un acuífero libre que tiene unas dimensiones aproximadas de $22 \mathrm{Km}^{2}$ y un espesor máximo de $60 \mathrm{~m}$ (ITGE-JUNTA, 1998). Está constituido por materiales de relleno: conglomerados, arenas, limos y arcillas (Morell et al. 1995) y su principal recarga se produce por infiltración de la lluvia útil y en menor medida por infiltración de aguas de escorrentía externas al acuífero ("arroyo de la Madre").

Este acuífero está conectado al acuífero subyacente de Sierra Gorda que ocupa una extensión de $293 \mathrm{Km}^{2}$. Se considera como esencialmente libre, aunque confinado en los bordes norte y este, y bajo el Polje de Zafarraya. El drenaje natural del acuífero del Polje hacia este acuífero de Sierra Gorda se altera sensiblemente por los bombeos realizados en unos 400 pozos de gran radio y escasa penetración que captan el acuífero detrítico en su sector central (ITGE-JUNTA, 1998).

Para evaluar el nivel de salinidad y nitratos del acuífero del Polje se muestrearon un total de diez pozos superficiales y diez parcelas comerciales en las que estaban ubicados dichos pozos (Figura 1). Tres de las parcelas tenían cultivado tomate, otras tres alcachofa y las restantes lechuga. El estudio se llevó a cabo desde mayo de 2015, momento en el que comenzaba la campaña de estos cultivos, hasta marzo de 2016, incluyendo así en el periodo de seguimiento la época de baldío. La selección de las parcelas se hizo en función de su ubicación geográfica y del cultivo plantado. De esta manera se consiguió una distribución representativa por toda la zona de influencia del acuífero y tener repetidas distintas parcelas con un mismo cultivo (Figura 2). 


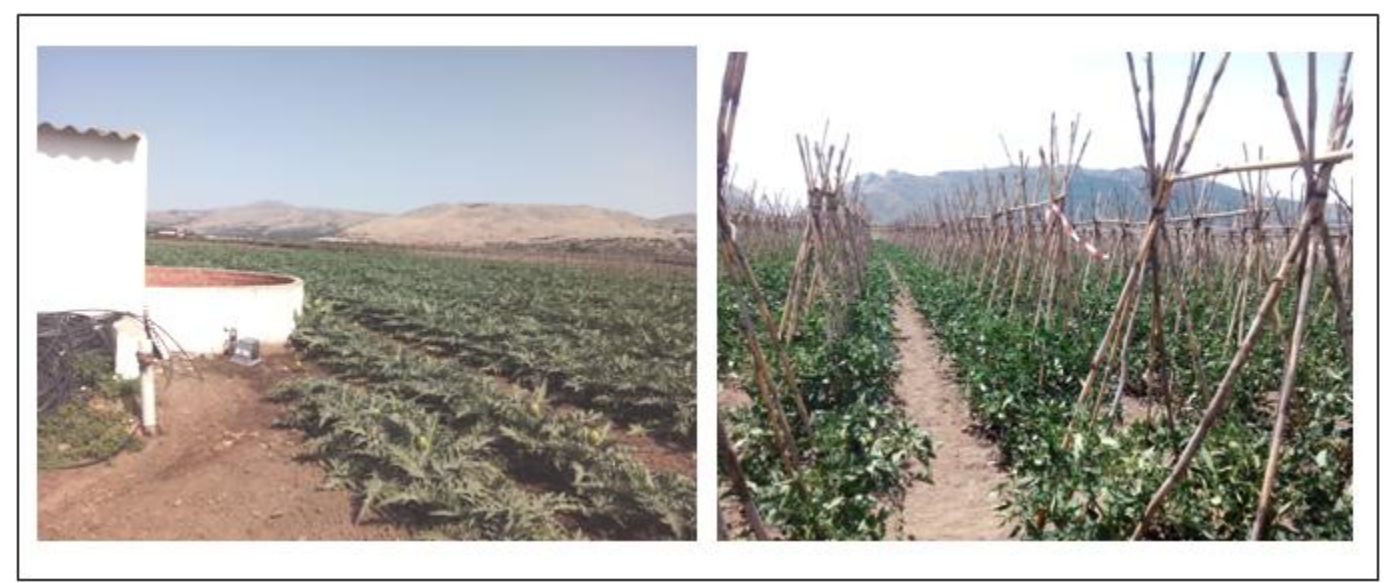

Figura 1. Vista de parcela experimental de alcachofa con pozo superficial (izq.). Detalle de parcela experimental de tomate (dcha.).

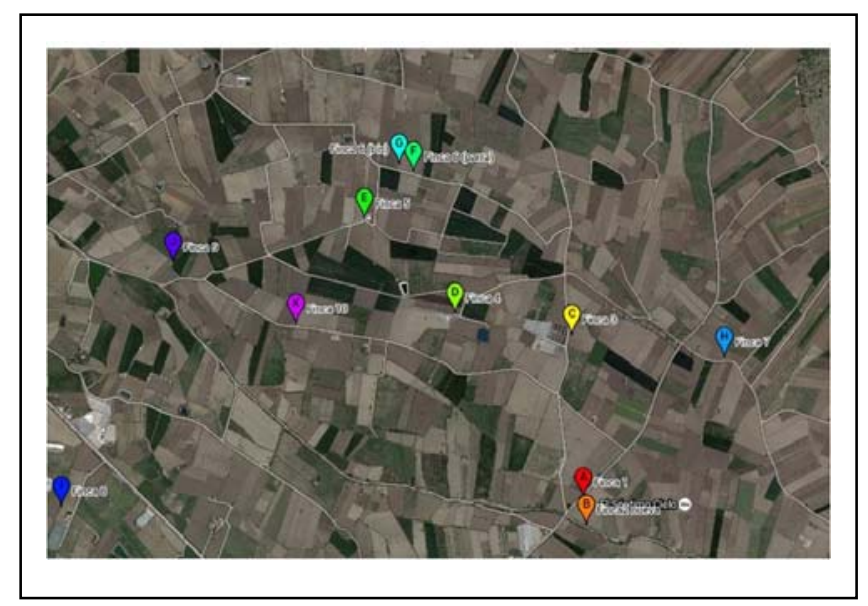

Figura 2. Distribución de las parcelas experimentales en el Llano de Zafarraya.

En cada parcela experimental se realizó un seguimiento de:

- La concentración de nitratos, [NO3] $\left(\mathrm{mg} \mathrm{L}^{-1}\right)$, y la conductividad eléctrica (CE) $\left(\mathrm{dS} \mathrm{m}^{-1}\right.$ ) del agua del pozo superficial. Para la obtención de muestras se empleó una botella de recogida de agua en profundidad. Estas muestras se recogían a 5 $\mathrm{m}$ respecto a la lámina de agua (Figura 3 ).

- La concentración de nitratos, [NO3-] $\left(\mathrm{mmol} \mathrm{L}^{-1}\right)$, y la conductividad eléctrica en la solución del suelo ( $\mathrm{CE}_{\mathrm{SS}}$ ) ( $\mathrm{dS} \mathrm{m}^{-1}$ ). Para la obtención de la solución del suelo se instalaron dos sondas de succión (Rhizon sampler SMS, Rhizosphere Research Products, NL-6700 AN Wageningen) en la zona radicular (Figura 3). 


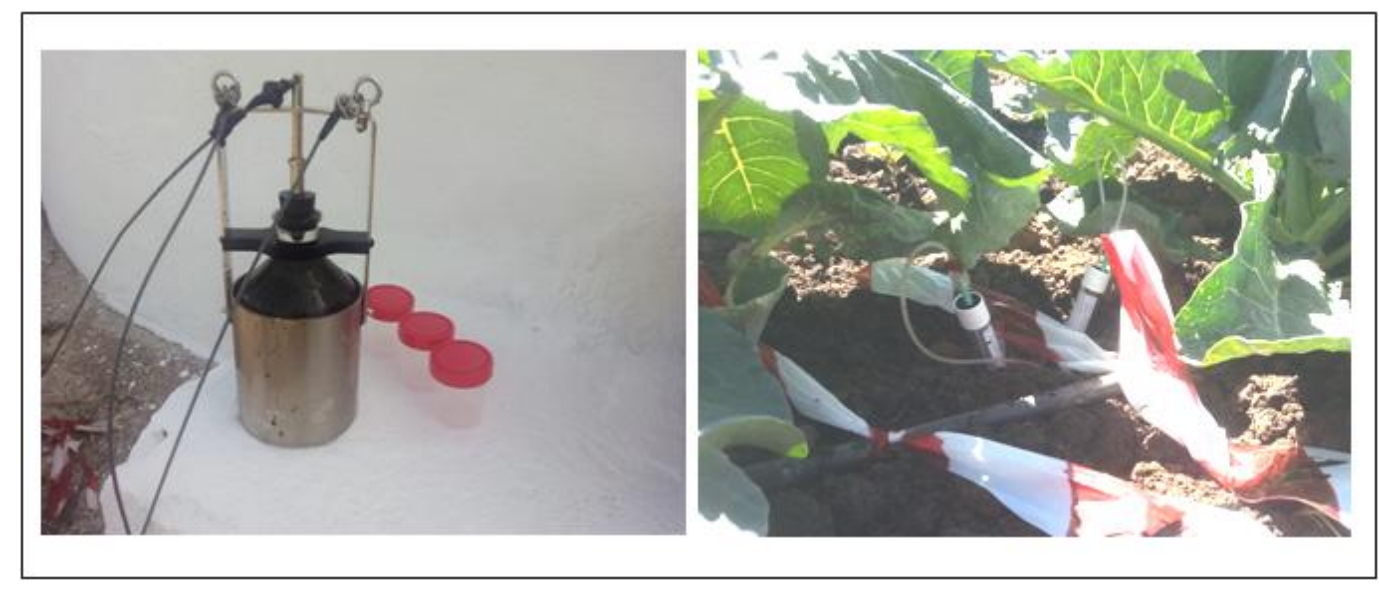

Figura 3. Botella para recogida de muestras de agua en profundidad (izq.).Sondas de succión tipo Rhizon instaladas en parcela experimental (drcha.).

Los análisis de [NO3-], se realizaron con el medidor de iones B-743 LAQUAtwin Compact Nitrate Meter (Horiba Co. Ltd., Japón). La CE con un conductímetro EC-Meter Basic 30+ (Crison Instruments). La periodicidad de los muestreos de las sondas de succión dependió del tipo de cultivo implantado y de la programación de los riegos de cada parcela experimental (semanal, quincenal, mensual). En el caso de los pozos el muestreo se realizó cada dos meses.

Destacar que el sistema de riego empleado en las parcelas de estudio es el riego localizado por goteo, comúnmente empleado en la zona. De manera generalizada la fertilización comienza con una aplicación previa en fondo de materia orgánica (estiércol) a razón de $0,5-0,7 \mathrm{~kg} \mathrm{~m}^{-2}$ o fertilizante organomineral peletizado (6-12-10) a una dosis de 0,3$0,4 \mathrm{~kg} \mathrm{~kg} \mathrm{~m}^{-2}$. Durante el ciclo de cultivo se realiza fertirrigación con fertilizantes sólidos solubles aplicados con tanques de fertilizante (abonadoras). Los equilibrios aplicados se deciden en función de la experiencia del agricultor o la recomendación del técnico asesor. Varían entre cultivos y ciclo y no se realiza un seguimiento en el suelo o el cultivo con el fin de corregir las recetas.

Los datos de pluviometría se obtuvieron de la estación meteorológica de Zafarraya (Latitud: 36 59' 25" N, longitud: 04 09' 13" W, altitud: 903 m).

\section{Resultados y discusión}

La conductividad eléctrica del agua de los pozos permaneció estable a lo largo de todo el periodo de muestreo (Figura 4). Los valores medios oscilaron entre 1,0 y $1,5 \mathrm{dS} \mathrm{m}^{-1}$. A pesar de la escasa variación se observa un descenso progresivo de la salinidad de manera que los niveles más elevados se obtuvieron entre mayo y julio $\mathrm{y}$, a partir de este momento se observó un ligero y progresivo descenso de la CE hasta el mes de enero (Figura 4). Estos valores de CE no representan problemas para los principales cultivos hortícolas que se plantan en la zona.

En lo que respecta a la concentración de nitratos, los resultados muestran niveles excesivos que oscilan entre 300 y $375 \mathrm{~g} \mathrm{~L}^{-1}$ (Figura 4). Estos valores están un 86\% por encima del umbral de $50 \mathrm{mg} \mathrm{L}^{-1}$ marcados por la directiva 96/6767CEE. La [NO3] no ha variado sustancialmente respecto a los observados por Morell et al. en 1995. Dichos autores obtienen valores máximos de $400 \mathrm{mg} \mathrm{L}^{-1}$. Aunque la situación de la [NO3-] no parece haberse agravado, estos datos ponen en evidencia la ausencia de planes de actuación para realizar una mejor gestión del riego y la fertilización en la zona, a pesar de la existencia de 
tecnologías para conseguirlo (máquinas de fertirriego, tensiómetros, sondas de succión, etc.).

Cabe destacar que la evolución de la [NO3-] a lo largo del periodo de estudio muestra un considerable aumento de los niveles de nitratos a partir del mes de julio, que está directamente relacionado con la fertilización de los cultivos (Figura 4). El descenso que se observa a partir de septiembre coincide con la presencia de lluvias (Figuras 4 y 5). Por tanto, aquí se observa un efecto de dilución de los nitratos en el agua de los pozos. Este fenómeno de dilución hace que los valores de nitratos obtenidos en el último mes de muestreo, marzo, sean similares a los de partida del mes de mayo, momento en el que empieza la campaña de cultivos (Figura 4). Morell et al. (1995) observan igualmente este considerable aumento a partir del mes de julio, aunque en su caso no lo relacionan con la fertilización de los meses previos sino que lo asocian al intenso proceso de lavado consecuente al periodo de lluvias. A la vista de los datos de precipitaciones obtenidos para este periodo de estudio esta no parece ser la causa (Figura 5).
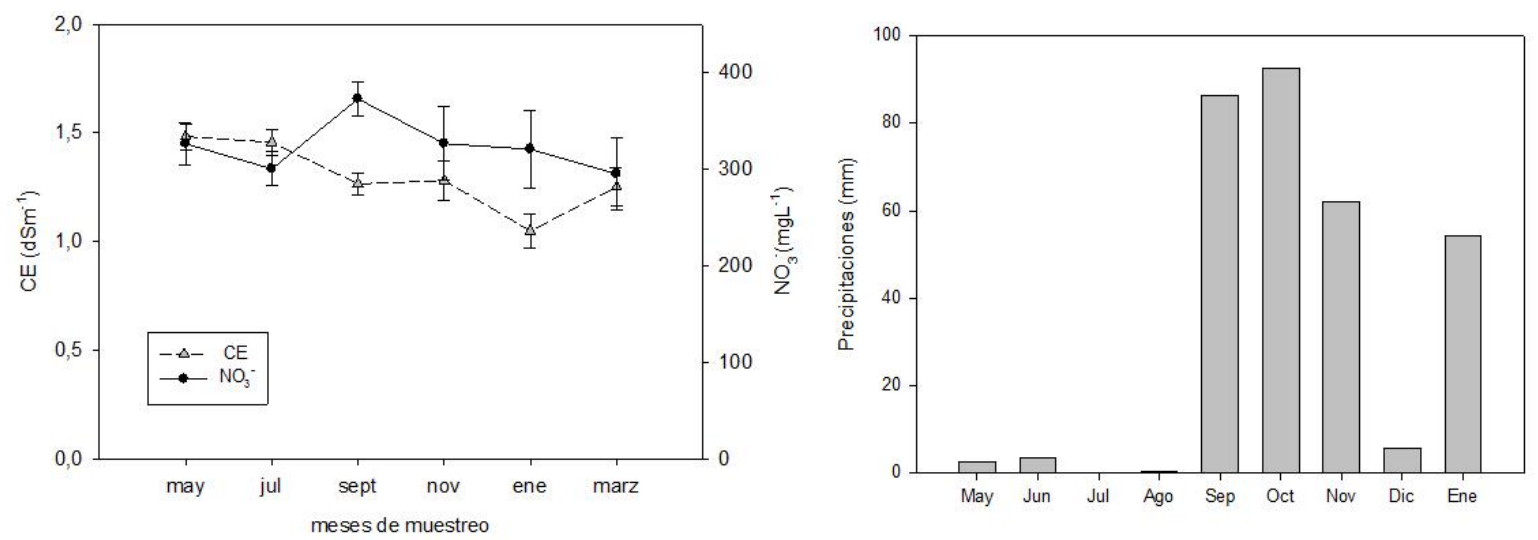

Figuras 4 y 5. Evolución de la concentración de nitratos [NO3-] y la conductividad eléctrica (CE) en los pozos del acuífero superficial detrítico (las barras verticales indican el error estándar). Precipitaciones correspondientes al periodo de estudio (mayo 2015-marzo 2016).

Los resultados de la conductividad eléctrica en la solución del suelo (CEss) en el cultivo de tomate muestran variabilidad entre parcelas y dentro de la misma parcela a lo largo del ciclo de cultivo. El rango de valores medios está entre 1,45 y $5 \mathrm{dS} \mathrm{m}^{-1}$ (Figura 6). A pesar de esta variabilidad, se observa que a comienzos del mes de agosto se produce un aumento de la CEss que se prolonga hasta la primera mitad del mes de septiembre, momento en el que empieza a descender (Figura 6). Estos valores parecen mostrar un sobreabonado de los cultivos en ese periodo, coincidiendo con el cuaje y engorde de los frutos.

La concentración de nitratos en la solución de suelo presenta una menor variabilidad y se aprecia una tendencia más clara a nivel de parcela y considerando el conjunto de parcelas. De esta manera, se observa que al comienzo del ciclo los valores medios de [NO3-] se encontraban dentro de un rango de $10-15 \mathrm{mmol} \mathrm{L}^{-1}$ y a partir de finales de junio se produce un aumento hasta alcanzar valores máximos en agosto de $27,5 \mathrm{mmol} \mathrm{L}^{-1}$ (Figura 7). Estos niveles están por encima de los niveles recomendables para este cultivo, 7-10 mmol $\mathrm{L}^{-1}$ (Fernández et al. 2012) y, por tanto, claramente por encima de las extracciones del cultivo. Su acumulación en el suelo a finales de campaña, coincidiendo con el periodo de lluvias, supone su lixiviación y la contaminación del acuífero del Polje. Estos resultados ponen de manifiesto la práctica habitual de aplicaciones excesivas de fertilizantes nitrogenados. 

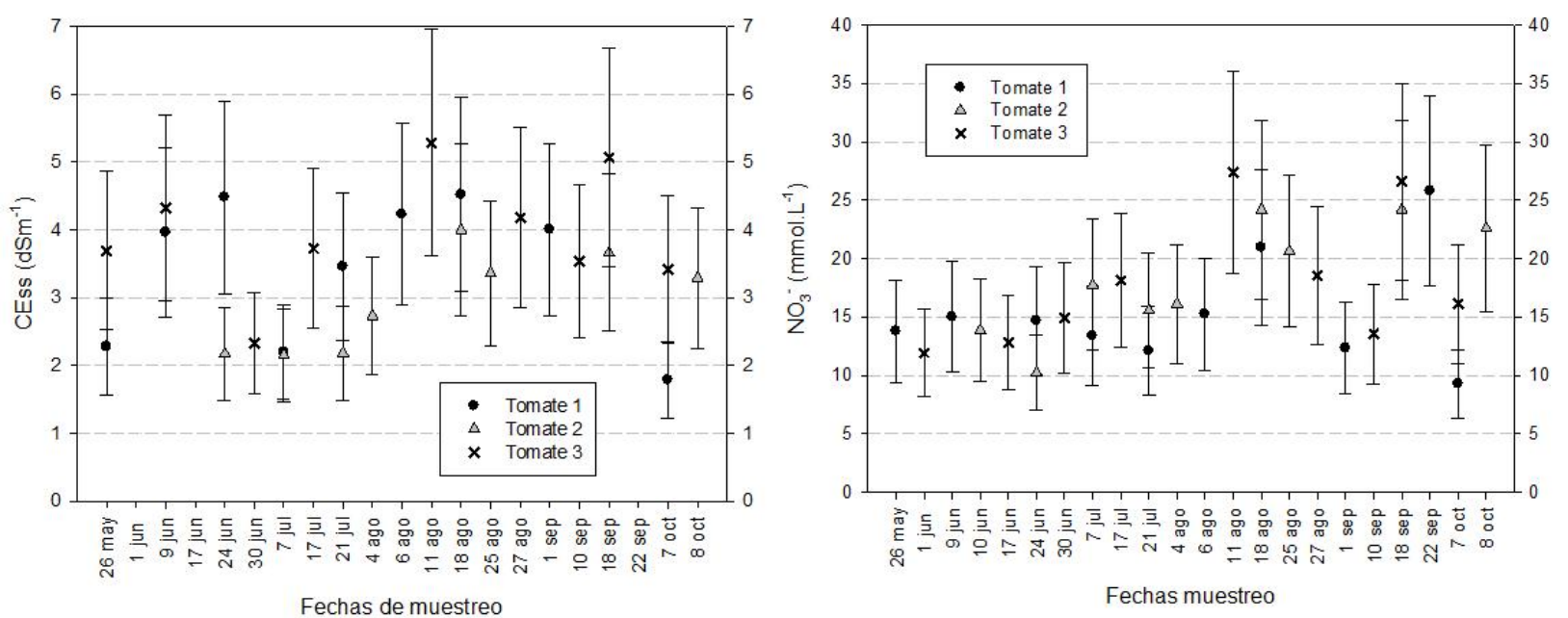

Figuras 6 y 7. Evolución de la conductividad eléctrica de la solución de suelo (CEss) y de la concentración de nitratos [NO3-] en parcelas experimentales de tomate

(las barras verticales indican el error estándar).

En las parcelas cultivadas de alcachofa la conductividad eléctrica en la solución del suelo (CEss) osciló entre 1 y $4 \mathrm{dS} \mathrm{m}^{-1}$ (Figura 8). Al comienzo del ciclo de cultivo la CEss presentaba valores en torno a 1-2 dS $\mathrm{m}^{-1}$ que se mantuvieron estables hasta finales de julio, momento a partir del cual se produjo un aumento alcanzando valores máximos en agosto. A finales de agosto se produce un descenso progresivo en los niveles de CEss situándose en valores similares a los observados al inicio del ciclo de cultivo (Figura 8). La [NO3'] de la solución del suelo es similar en todas las parcelas de alcachofa en el periodo de mayo-julio. Los valores para este periodo estuvieron entre 3,5 y $11 \mathrm{mmol} \mathrm{L}^{-1}$ (Figura 9). A partir de julio una de las parcelas mostró un aumento significativo alcanzando niveles superiores a 20 $\mathrm{mmol} \mathrm{L}^{-1}$, mientras que las otras dos permanecieron con valores muy similares durante todo el ciclo de cultivo (Figura 9).
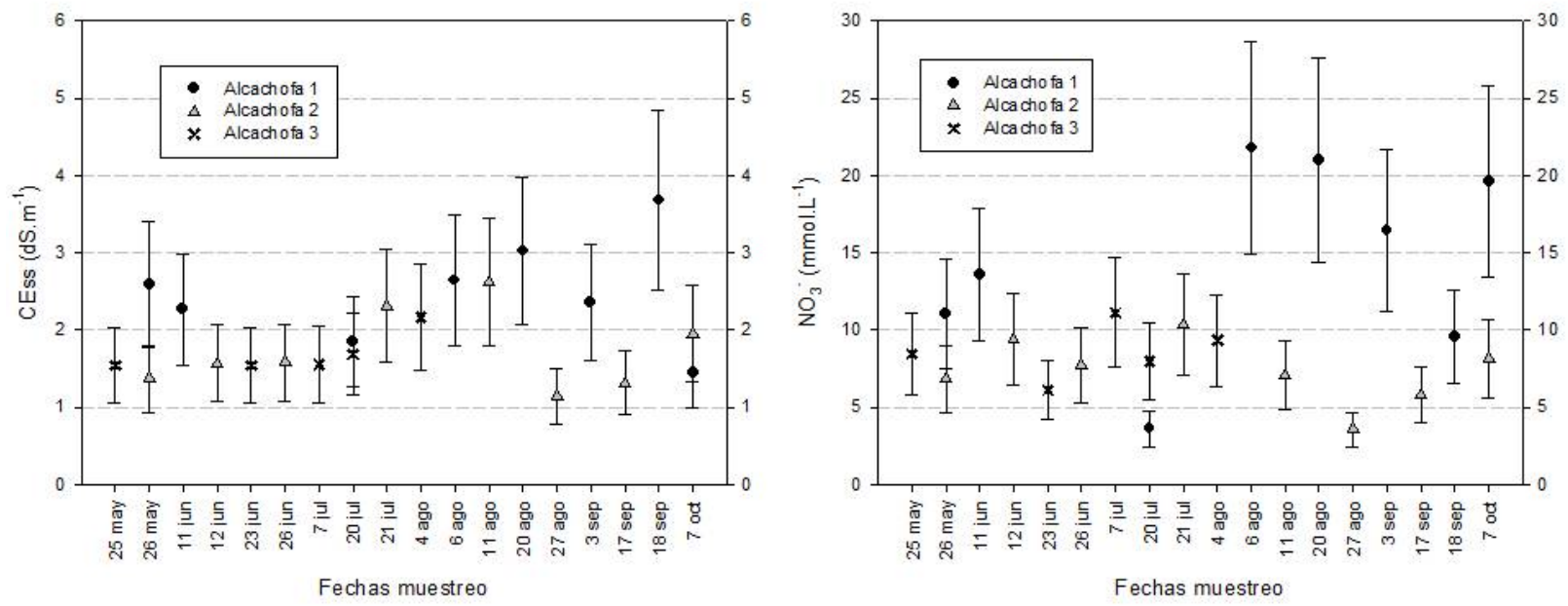

Figuras 8 y 9. Evolución de la conductividad eléctrica de la solución de suelo (CEss) y de la concentración de nitratos [NO3-] en parcelas experimentales de alcachofa (las barras verticales indican el error estándar).

Los resultados obtenidos en las parcelas cultivadas con lechuga no se muestran en este trabajo por la variabilidad los datos obtenidos. 


\section{Conclusiones y recomendaciones}

Los niveles de salinidad obtenidos en el agua de los pozos a lo largo del periodo de estudio oscilaron entre 1,0 y $1,5 \mathrm{dS} \mathrm{m}^{-1}$. Estos valores no representan problemas para los principales cultivos hortícolas que se plantan en la zona. Sin embargo, en lo que respecta a la concentración de nitratos, los resultados muestran niveles excesivos que superan en un $86 \%$ el umbral de $50 \mathrm{mg} \mathrm{L}^{-1}$ marcado por la directiva 96/6767CEE.

Los niveles de nitratos en la solución del suelo a partir del mes de junio están claramente por encima de las extracciones del cultivo, sobre todo en el cultivo de tomate. Su acumulación en el suelo a finales de campaña, coincidiendo con el periodo de lluvias, suponen su lixiviación y la contaminación del acuífero del Polje. Estos resultados ponen de manifiesto la práctica habitual de aplicaciones excesivas de fertilizantes nitrogenados.

La recomendación inicial sería mantener una concentración de nitratos estable a lo largo del ciclo de cultivo para evitar acumulaciones en el suelo y sobre todo al final del mismo (Fernández et al. 2011). En tomate, cultivo donde se han obtenido los resultados más negativos de nitratos en solución de suelo, se recomienda mantener los niveles entre 7 y 10 $\mathrm{mmol} \mathrm{L}^{-1} \mathrm{e}$, incluso, reducirlos al final del ciclo para minimizar la lixiviación asociada a las Iluvias otoñales (Fernández et al. 2012). Este control y ajuste es posible empleando sondas de succión y análisis rápido de nitratos a lo largo de todo el ciclo de cultivo.

\section{Bibliografía}

Cherif, L., Pulido-Bosch, A., López Chicano, M., Morell, I. \& Gámez, J.A. (1995). Las actividades agrícolas en el Polje de Zafarraya y la evolución del contenido en nitratos en dos manantiales del acuífero de Sierra Gorda (Granada y Málaga). Geogaceta, 18, 146-149.

Decreto 36/2008, 5 de febrero. BOJA núm. 36 de 20 de febrero 2008 en el que se designan las zonas vulnerables y se designan medidas contra la contaminación por nitratos de origen agrario.

Fernández, M., Baeza, R., Cánovas, G. \& Martín, E. (2011). Protocolo de actuación para disminuir la contaminación por nitratos en cultivos de pimiento y tomate bajo abrigo.17pp.

Fernández, M., Martín, E., Cánovas, G. \& Baeza, R. (2012). Manejo controlado de la aplicación de nitratos en cultivos de tomate y pimiento en invernadero. Vida Rural, 343, 38-44.

Gómez-Zotano, J., Alcántara-Manzanares, J., Olmedo-Cobo, J.A. \& Martínez-lbarra, E. (2015). La sistematización del clima mediterráneo: identificación, clasificación y caracterización climática de Andalucía (España). Revista de Geografía Norte Grande, 61, 161-180.

ITGE-JUNTA, D.A. (1998). Atlas hidrogeológico de Andalucía. Consejería de Obras Públicas y Transportes, Consejería de Trabajo e Industria de la Junta de Andalucía, Instituto Tecnológico Geominero de España, 216 pp. Madrid.

Morell, I., López-Chicano, M., Pulido-Bosch, A., Gámez, J.A. \& Cherif, L. (1995). Procesos de lixiviado de nitratos en el acuífero detrítico del polje de Zafarraya (Granada). Avances en la investigación en zona no saturada, 63-69. 
XXXYDIV Congreso Nacional de Riegos, Sevilla 2016

Orden del 18 de noviembre de 2008, BOJA nº del 8 de enero de 2009, por la que se aprueba el programa de actuación aplicable en las zonas vulnerables a la contaminación por nitratos en Andalucía. 